310 Parcus: Ueber einige nene Gehirnstoffe.

innern, dass der Schmelzpunkt der Säure nicht bei $+16,55^{\circ}$, sondern bei $+7,45^{0}$ liegt.

Die beiden Quantitäten der Hydrate, welche chemisch homolog und äquivalent sind:

$$
\mathrm{HCOOH}=46 \text { und } \mathrm{CH}_{3} \mathrm{COOH}=60 \text {, }
$$

zeigen also auch in ihrem physischen $\nabla$ erhalten die folgende Uebereinstimmung:

Die Volume derselben im Dampfaustand sind bei derselben Temperatur ${ }^{1}$ ) und demselben Druck gleich gross (Avogadro's Gesetz).

Sie entwickeln beim Festwerden dieselbe Wärmemenge (s. diese Untersuchung).

Ihr Volum zieht sich beim Festwerden relativ gleich viel zusammen (s. diese Untersuchung).

Ihre Ausdehnung beim Erwärmen ist wenigstens in der Näbe des Schmelzpunktes proportional (s. diese Untersuchung).

\title{
Ueber einige nene Gehirnstoffe;
}

von

\section{Eugen Parcus.}

Im Jahre 1792 erhielt Foureroy ${ }^{2}$ ) durch Extraction des Gehirns mit heissem Alkohol eine Lösung, welche beim Erkalten eine in Blättern krystallisirende Substanz ausschied.

Dieser alkoholische Auszug, welcher ausser Cholesterin die interessantesten chemischen Verbindungen des Gehirns: das Lecithin, Protagon und Cerebrin gelöst enthält, ist seitdem der Ausgangspunkt so mancher mühevoller Untersuchungen hervorragender Forscher gewesen, ohne dass indessen die daraus hervorgegangenen Resultate unsere Kenntnisse von diesen Stoffen erheblich erweitert hätten.

1) Wenn dieselbe hinreichend hoch ist.

2) Ann. Chim. Phys. 16, 282. 
Parcus: Ueber einige neue Gehirnstoffe. 311

Während jedoch Lecithin ${ }^{1}$ ) aus dem Gehirn rein dargestellt wurde und sein Vorkommen in Eiern, aus welchen es leichter isolirt werden kann, zu seiner Erforschung wesentlich beitrug; während es sogar in neuester Zeit gelungen ist, das von Liebreich ${ }^{2}$ ) entdeckte Protagon, das lange Zeit für ein Gemenge von Cerebrin mit Lecithin gehalten wurde, rein darzustellen, scheint ein Gleiches nicht mit dem Cerebrin der Fall gewesen zu sein.

$M \ddot{u l l e r}{ }^{3}$ ), welcher das Cerebrin zuerst phosphorfrei erhielt, giebt zwar an, durch Umkrystallisiren aus Alkohol vollkommen reines Cerebrin dargestellt zu haben, doch wichen spätere, wenn auch noch so dürftige Angaben anderer Forscher über die Zusammensetzung des Cerebrins von den seinigen so bedeutend ab, dass es der Mühe werth erschien, diese Frage auf's Neue zum Gegenstande einer eingehenden Untersuchung zu machen.

Unbekannt mit Lecithin und der Thatsache seiner Zersetzung durch Kochen mit Alkohol, bedienten sich ältere Forscher, wie Fourcroy, zur Darstellung der Gehirnstoffe dieses Lösungsmittels bei der Siedetemperatur. Da sie gleichzeitig eine gründliche Reinigung der aus dem erkalteten Auszuge ausgefallenen Substanzen vorzunehmen unterliessen, so stellten die von ihnen beschriebenen Körper ein Gemenge von Cerebrin, Protagon, Cholesterin und Lecithinresten dar, in welchem die beiden erstgenannten Stoffe die vorherrschenden Bestandtheile bildeten.

Hierher gehören: Vauquelin's ${ }^{4}$ ) weisse und rothe fette Materie; ebenso Couerbe's ${ }^{5}$ ) Cerebrot, Cephalot, Stearoconot und Eléencephol.

Fremy $\left.{ }^{6}\right)$ wendete zwar dieselbe Methode an, doch ist seine Cerebriusäure jedenfalls eine reinere Substanz, als Vauquelin's und Couerbe's Körper. Dieses Produkt,

1) Diaconow, Med. Centralbl. 1868.

2) Ann. Chem. Pharm. 134, 29 (1865).

3) Das. 105, 361.

4) Ann. Chim. Phys. 81, 37 (1812).

5) Das. [2] 56, 164 (1834).

$\left.{ }^{6}\right)$ Journ. de Pharm. 27, 453 (1840). 
312 Parcus: Ueber einige neue Gehirnstoffe.

welches von v. Bibra und von W. Müller') für Cerebrin gehalten wurde, hat nichts mit letzterem gemein, besitzt dagegen eine grosse Aehnlichkeit mit Liebreich's ? Protagon und stimmt bezüglich seiner Zusammensetzung mit dem Protagon Gamgee's und Blankenhorn's ${ }^{3}$ ) überein.

\begin{tabular}{|c|c|c|c|}
\hline & $\begin{array}{c}\text { Liebreich's } \\
\text { Protagon. } \\
\text { Mittel. }\end{array}$ & $\begin{array}{c}\text { Gamgee's u. Blanken- } \\
\text { horn's Protagon. } \\
\text { Mittel. }\end{array}$ & $\begin{array}{c}\text { Frem } y^{2} s \\
\text { Cerebrinsäure. }\end{array}$ \\
\hline $\mathrm{C}$ & $=66,74$ & 66,39 & 66,7 \\
\hline $\mathrm{H}$ & $=11,74$ & 10,69 & 10,6 \\
\hline $\mathrm{N}$ & 2,80 & 2,39 & 2,3 \\
\hline $\mathbf{P}$ & 1,23 & 1,068 & 0,9 \\
\hline 0 & $=17,40$ & 19,43 & 19,5 \\
\hline
\end{tabular}

Mit Protagon theilt Fremy's Cerebrinsäure weiter die Eigenschaft, erst bei hoher Temperatur unter Zersetzung zu schmelzen. $\mathrm{Ob}$ sie jedoch mit dem Protagon als identisch anzusehen ist, lässt sich vorerst nicht entscheiden. Zunächst widerspricht dieser Auffassung die Art der Darstellung, wie sie Fremy anwandte. Derselbe kochte das Gehirn mit absolutem Alkohol, um ihm Wasser zu entziehen und die Albuminstoffe zu coaguliren. Das Coagulum wurde mit heissem Aether extrahirt, welcher abdestillirt die Cerebrinsäure zurückliess. Diese wurde in heissem, mit Schwefelsäure schwach angesänertem Alkohol gelöst, und das beim Erkalten Ausgeschiedene zur weiteren Reinigung mehrmals aus kochendem Aether umkrystallisirt. Es ist daher möglich, dass seine Cerebrinsäure nur aus Zersetzungsprodukten des Protagons bestand. Diese Ansicht wird durch die Thatsache unterstützt, dass Protagon ${ }^{4}$ ) durch Kochen mit Aether eine Zersetzung erleidet; im vorliegenden Falle jedoch dürfte dieselbe nur von geringem Belang sein, da zu jener Zersetzung 15 stündiges Kochen erforderlich ist. ${ }^{5}$ )

Wenn es sich daher aus weiteren Untersuchungen er-

1) Ann. Chem. Pharm. 105, 361.

2) Das. 134, 29 (1865).

3) Hoppe-Seyler's Ztschr. 3, 260 (1879).

4) Gamgee u. Blankenhorn, a. a. O. S. 280.

5) Das. S. 280. 
giebt, dass Protagon durch kurzes Kochen in seinen Lösungsmitteln nicht verändert wird, so gewinnt die Ansicht der Identität der Cerebrinsäure Fremy's mit dem Protagon Gamgee's und Blankenhorn's an Gewicht. Fremy giebt weiter an, Salze der Cerebrinsäure erhalten zu haben. Das Barytsalz hat er analysirt. Nach Liebreich ${ }^{1}$ ) wird Protagon durch Kochen mit Barytwasser zersetzt. Wendet man letzteres auf die Cerebrinsüure Fremy's an, so beging Fremy nur den Fehler, die gebildeten Salze für Salze der Cerebrinsäure anzusehen. v. Bibra setzte die Untersuchungen über die Cerebrinsäure Fremy's fort, doch machte er nicht den ätherischen, sondern den alkoholischen Auszug des Gehirns zum Ausgangspunkte seiner Untersuchung, wodurch er eine von Fremy's Cerebrinsäure abweichende Substanz erhalten musste. ${ }^{2}$ ) Diese Substanz kochte er mit Kali, zersetzte das gebildete cerebrinsaure Kali mit Salzsäure und erhielt durch Wiederholung dieser Operationen reine Cerebrinsäure. Diese besass jedoch nicht die Eigenschaft, mit Basen Verbindungen einzugehen, und widerstand ziemlich lange der Einwirkung concentrirter Säuren.

Geht schon aus diesem Verhalten hervor, dass v. Bibra's Cerebrinsäure eine widerstandsfähigere Substanz als die Cerebrinsäure Fremy's war, so weisen auch alle sonstigen Eigenschaften derselben darauf hin, dass sie ein durch phosphorhaltige Körper verunreinigtes Cerebrin darstellte. Es ist daher merkwürdig, dass die Analysen derselben fast dieselben Resultate wie Fremy's Cerebrinsäure lieferten.

\begin{tabular}{|c|c|c|}
\hline Fremy's & Cerebrinsäure. & v. Bibra's Cerebrinsäure. \\
\hline $\mathrm{C}$ & $=66,7$ & 66,79 \\
\hline $\mathrm{H}$ & $=10,6$ & 10,65 \\
\hline $\mathbf{N}$ & 2,3 & 2,51 \\
\hline $\mathbf{P}$ & 0,9 & 0,52 \\
\hline 0 & $=19,5$ & 19,52 \\
\hline
\end{tabular}

1) Ann. Chem. Pharm. 134, 29.

$\left.{ }^{2}\right)$ Die Angaben über die v. Bibra'sche Cerebrinsäure habe ich, da ich der Originalabhandlung nicht habhaft werden konnte, der Arbeit W. Müller's: Ueber die chemischen Bestandtheile des Gehirns (Ann. Chem. Pharm. 105, 361), welche sich mit Ausführlichkeit über die v. Bibra'sche Cerebrinsäure verbleitet, entnommen. 
314 Parcus: Ueber einige neue Gehirnstoffe.

Auch Müller ${ }^{1}$ ) ging in seiner Untersuchung von der Cerebrinsäure Fremy's aus. Er wich aber bedeutend von der Darstellungsweise seiner Vorgänger ab, indem er, Liebig nachahmend, das Gehirn vor der Extraction mit Alkohol mit Barytwasser anrieb und zum Sieden erhitzte. Er erreichte durch diese Methode unbewusst die Entfernung des damals noch unbekannten Lecithins, welches bei dieser Operation in seine Componenten zerfällt, die mit Ausnahme des Neurins an Baryt gebunden werden. Ein Theil der entstandenen Produkte geht in das basische Filtrat über, während ein anderer Theil bei der weiter vorzunehmenden Extraction mit kochendem Alkohol ungelöst zurückbleibt. Müller erhielt beim Erkalten dieses alkoholischen Auszuges einen voluminösen, weissen Niederschlag, welcher, mit kaltem Aether erschöpfend ausgezogen, einen gelblichen Rückstand hinterliess, der beim wiederholten Umkrystallisiren aus kochendem Alkohol eine seiner Angabe nach vollkommen reine Substanz lieferte.

Da dieselbe in ihren Lösungen neutral reagirte und auch sonst keine Eigenschaften besass, welche sie als eine Säure charakterisirten, nannte er sie Cerebrin und stellte gestützt auf einige Elementaranalysen, welche im Durchschnitt $68,45 \% \mathrm{C}, 11,20 \% \mathrm{H}$ und $4,6 \% \mathrm{~N}$ lieferten, für sie die Formel $\mathrm{C}_{34} \mathrm{H}_{33} \mathrm{NO}_{6}$ auf. Dieses Cerebrin besitzt die Eigenschaften, wie sie bis in die neneste Zeit in verschiedenen Lehrbüchern angeführt worden sind.

Seit Müller, dessen Arbeit im Jahre 1858 erschien, ist nur selten der Versuch gemacht worden, das Cerebrin aus der Gehirnmasse zu isoliren, es fanden sich daher nur wenige weitere Angaben über diesen Körper vor.

Liebreich ${ }^{2}$ ) hielt das Cerebrin für ein Zersetzungsprodukt seines Protagons; es gelang ihm aber nicht, in den durch Kochen mit Barytwasser hervorgegangenen Spaltungsprodukten des Protagons Cerebrin nachzuweisen.

1) Ann. Chem. Pharm. 105, 361.

2) Ann. Chem. Pharm. 134, 42. 
Parcus: Ueber einige neue Gehirnstoffe. 315

Andere ${ }^{1}$ ) erhielten, wie Mü1ler, phosphorfreies Cerebrin, fanden aber weniger Stickstoff als dieser.

Otto und Köhler²) gaben sogar an, stickstofffreie Cerebrine dargestellt zu haben; die von ihnen beschriebenen Stoffe sind jedoch nicht identisch.

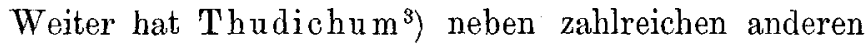
Körpern vier phosphorfreie, stickstoffhaltige Substanzen aus dem Gehirn erhalten, welche er Cerebrin, Stearoconot, Kerasin und Phrenosin benennt, und für welche er folgende Formeln aufstellt:

$$
\begin{aligned}
& \text { Cerebrin } \\
& \text { Stearoconot } \\
& \text { Phrenosin } \mathrm{C}_{34} \mathrm{C}_{67} \mathrm{H}_{68} \mathrm{NO}_{2} \mathrm{O}_{8} \\
& \text { Kerasin } \mathrm{C}_{46} \mathrm{H}_{91} \mathrm{NO}_{9} .
\end{aligned}
$$

Von Zersetzungen des Cerebrins war nur bekannt, dass es durch Kochen mit Säuren eine Lösung giebt, welche alkalisches Kupferoxyd reducirt. ${ }^{4}$ ) Dieses Verhalten, sowie die Fähigkeit dieser Lösungen, die Ebene des polarisirten Lichtes zu drehen, hat zu der Annahme veranlasst, dass das Cerebrin ein Glucosid sei. War es daher schon von grossem Interesse, den Nachweis zu führen, ob Cerebrin Stickstoff enthalte oder stickstofffrei sei (Köhler und Otto), so wurde dasselbe durch die Aussicht noch wesentlich gesteigert, der Reindarstellû́ng des Cerebrins das Studium der Zersetzungsprodukte folgen lassen zu können. $\left.{ }^{5}\right) \mathrm{Da}$ Müller sein Cere-

1) Bourgoin, Bull. Soc. chim. 21, 482.

2) Virchow's Archiv 41, 26 כ.

3) Chemical news 31, 112.

$\left.{ }^{4}\right)$ Diaconow, Med. Centralbl. 1868, No. 7.

5) Die Spaltung des Cerebrins ist in der. Folge von Geoghegan vorgenommen worden. Derselbe erhielt durch Behandeln des Cerebrins mit concentrirter Schwefelsäure und Fällen der crhaltenen Lösung mit Wasser eine Kupferoxyd reducirende saure Lösung und eine stickstofffreie, in Aether lösliche Substanz, welche er Cetylid nannte. Das letztere zersetzt sich beim Schmelzen mit Aetzkali unter Eutwickelung von Wasserstoff nnd Methan. Aus dem Rückstande erhielt derselbe durch Ansäuern mit Salzsäure und Extrahiren mit Aether eine Substanz, welche, aus Alkohol umkrystallisirt, beim Abkühlen in ge- 
316 Parcus: Ueber einige neue Gehirnstoffe.

brin erst durch mehrmaliges Umkrystallisiren ganz rein erhalten hatte, lag es nahe, darauf $\mathrm{zu}$ achten, was für eine Substanz in der alkoholischen Mutterlauge gelöst bleiben würde.

\section{Voruntersuchung.}

Das Cerebrin wurde nach der Methode von Müller dargestellt. Ochsengehirn, von Blut und Membranen befreit, wurde durch Aufkochen mit Barythydrat coagulirt, das Coagulum mit kaltem Alkohol und Aether behandelt und dann mit absolutem Alkohol ausgekocht. Beim Erkalten fiel eine beträchtliche Menge eines flockigen Niederschlages aus, welcher getrocknet und verbrannt einen Rückstand hinterliess, in welchem Phosphor nachgewiesen werden komte. Dieser Niederschlag wurde mehrmals mit kaltem Aether behandelt und dann aus Alkohol umkrystallisirt. Das so gereinigte Cerebrin zeigte sich weisser und aschefreier, als das früher erhaltene; in Wasser erhitzt quoll es auf. Natronlauge und concentrirte Säure verhinderten diese Quellung.

Beim Umkrystallisiren war ein Rückstand geblieben, welcher sich nur bei anhaltendem Kochen in Alkohol löste und in heissem Wasser unverändert blieb. Er erwies sich als ein Barytsalz, dessen Säure, der Fettsäurereihe angehörig, wahrscheinlich Stearinsäure war.

Die Ausbeute an Cerebrin war eine geringe. Aus drei Gehirnen erhielt ich nur wenige Gramme, welche beim Umkrystallisiren noch beträchtliche Mengen in der Mutterlauge zurückliessen. Aus diesen fiel nach 1-2 Tagen, manchmal

wundenen blättrigen Krystallen ausfiel. Der Schmelzpunkt dieser Substanz, sowie das Barytsalz, welches dieselba lieferte, fübrte ihn zu dem Schluss, dass er es mit Palmitinsäure zu thun habe. Das Auftreten von Wasserstoff liess ihn vermuthen, dass die Palmitinsâure aus Cetylalkohol hervorgegangen sei, und diesor Alkohol wahrscheinlich in Verbindung mit einem Kohlehydrat darin enthalten sei, wofür ihm das entweichende Grubengas zu sprechen schien. Der durch Behandeln mit concentrirter Schwefelsäure abgespaltene Stickstoff war zum Theil in Gestalt von Platinsalmiak dureh Zusatz von Platinchlorid zur schwefelsauren Lösung nachweisbar; die Kupferlösung reducirende Substanz hielt er für eine Säure. 
Parcus: Ueber einige neue Gehirnstoffe. 317 anch kurz nachdem abfiltrirt worden war, eine flockige gallertartige Masse nieder.

Die Analysen des Cerebrins lieferten bezüglich des Kohlenstoffs und Wasserstoffs unbefriedigende, in weiten Grenzen schwankende Resultate. Ich erhielt:

$\begin{array}{ll}69,25 \% \mathrm{C} & 12,10 \% \mathrm{H} \\ 68,65 ", & 12,07, ", \\ 68,22 ", & 11,06 ", " \\ 69,35 ", & 12,29, ", \\ 70,17 ", " & 12,34, ", \\ 70,35, " & 11,79, ",\end{array}$

Dagegen stimmten die Stickstoffbestimmungen besser überein. Sie ergaben:

$$
\begin{aligned}
& 2,63 \% \mathrm{~N} \\
& 2,61 " n \\
& 2,63 ",
\end{aligned}
$$

also $2 \%$ weniger als Müller gefunden hatte. Bei weiteren Analysen wurden einmal $3,01 \% \mathrm{~N}$, dann, nachdem häufiger umkrystallisirt worden war,

erhalten.

$$
\begin{aligned}
& 2,16 \% \mathrm{~N} \\
& 2,36 ", \\
& 2,03 ", \\
& 2,26 ",
\end{aligned}
$$

Das Cerebrin löste sich in Methylalkohol, Schwefelkohlenstoff, Chloroform und Benzol leicht in der Wärme und fiel daraus beim Verdunsten in mikroskopischen Kugeln nieder. Von Chloroform aufgenommen bildete es beim Abkühlen einen wachsartigen, durchscheinenden Kuchen, welcher beim Erhitzen vollständig schmolz, ohne der Hinzufügung von mehr Chloroform zu bedürfen. Das Cerebrin zeigte sonst alle die Eigenschaften, wie sie von Müller angegeben worden waren.

Durch Behandeln des Cerebrins mit absolutem Alkohol bei $40^{\circ}$ erhielt ich eine Flüssigkeit, welche beim Erkalten zu einer Gallerte erstarrte. Der von siedendem Alkohol aufgenommene Rückstand fiel beim Erkalten seiner Lösung in Pulverform nieder.

Es geht daraus hervor, dass das Cerebrin ein Gemenge 
318 Parcus: Ueber einige neue Gehirnstofte.

von zwei Körpern gewesen ist. Die Voruntersuchung hatte demnach folgende Resultate geliefert:

1) Das Cerebrin enthält etwas über $2 \% \mathrm{~N}$.

2) Beim Umkrystallisiren des Cerebrins bleibt eine Substanz in der Mutterlauge zurück, welche die Eigenschaft besitzt, sich aus concentrirten heissen Lösungen beim Erkalten als Gallerte auszuscheiden.

\section{Eigentliche Untersuchung.}

Zur weiteren Verfolgung der in der Voruntersuchung gefundenen Resultate war die Darstellung grösserer Mengen Substanz erforderlich.

Es wurde hierbei folgendermaassen verfahren:

Das von Membranen und Blut befreite Ochsengehirn wurde mehrfach mit kaltem Wasser ausgewaschen, dann durch einen leinenen Sack gepresst und mit concentrirtem Barytwasser unter Umschütteln zum einmaligen Aufkochen erhitzt. Hierauf liess ich das Ganze einige Zeit stehen, bis sich das Coagulum zu Boden gesetzt hatte. War die überstehende Lösung klar, so konnte die Operation als beendet angesehen werden; war sie dagegen trübe, so musste nach Zusatz von einer weiteren Menge Barythydrat noch einmal aufgekocht werden. Dann wurde filtrirt, der Niederschlag mit heissem, fast kochendem Wasser ausgewaschen, alsdann getrocknet und im Kolben mit absolutem Alkohol am Rückflusskühler unter fortwährendem Umschütteln erhitzt, heiss filtrirt und der Rückstand in derselben Weise noch 4-5 Mal extrahirt. Der erste Auszug schäumte gewöhnlich sehr stark, schied aber beim Erkalten wenig aus. Erst aus den folgenden Auszügen fiel das Cerebrin beim Erkalten reichlicher nieder, aber noch mit Cholesterin verunreinigt. Um die Hauptmenge dieser letzteren Substanz zu entfernen, wurde das Cerebrin einmal mit grossen Mengen kalten Aethers, sodann aber in Drechsel's Extractionsapparat mit heissem Aether erschöpft, nachdem ich mich vorher überzeugt hatte, dass auf diese Weise nur verbältnissmässig geringe Mengen von Cerebrin gelöst werden. Das rohe Cerebrin, welches eine klebrige Beschaffenheit besessen hatte, wurde nach mehr- 
Parcus: Ueber einige neue Gehirnstoffe. 319

tägigem Behandeln mit heissem Aether staubtrocken und pulverisirbar. Die ersten ätherischen Auszüge lieferten sebr ansehnliche Mengen von Cholesterin; die späteren waren cholesterinfrei, setzten aber beim Erkalten weisse, flockige Niederschläge $a b$. Da ich dieselben für Cerebrin hielt, wurde das Extrahiren mit heissem Aether aufgegeben.

Auf diese Weise verarbeitete ich über 90 Ochsengehirne, sie lieferten etwa $250 \mathrm{Grm}$. Rohcerebrin. Dieses war stark aschehaltig, aber phosphorfrei.

Das Roheerebrin wurde einmal aus Alkohol bei $60^{\circ}$ unkkrystallisirt, um das bei der Voruntersuchung erwähnte, ungelöst bleibende Barytsalz zu entfernen. Der Rückstand enthält stets noch Cerebrin. Um dieses zu gewinnen, kocht man den Rückstand mit Alkohol und behandelt den aus der abgekühlten, alkoholischen Lösung erhaltenen Niederschlag so lange mit Alkohol bei $60^{\circ}$, als sich noch etwas löst.

In der Mutterlauge zeigte sich die gallertartige Ausscheidung wieder. Um dieses löslichere Prodult rascher zu entfernen, wurde die ganze Menge des Cerebrins aus grossen Quantitäten Alkohol umkrystallisirt. Die dabei gewonnenen Mutterlaugen liess ich zwei Tage stehen; dann erst wurde die ausgeschiedene Gallerte von der Flüssigkeit getrennt. Das bis auf einen geringen Rest abdestillirte Filtrat hinterliess eine gelbe Lösung, welche beim Erkalten ansehnliche Mengen eines weissen, flockigen Körpers ausschied. Diesen will ich der Kürze halber mit $\mathbf{D}$ und die aus der Mutterlauge sich absetzende Gallerte mit M. bezeichnen. Das zweimal umkrystallisirte Cerebrin wurde, nachdem es im Vacuum über Schwefelsäure getrocknet worden war, analysirt. Die Verbrennungen wurden mit Kupferoxyd und metallischem Kupfer ausgeführt. Der Stickstoffgehalt wurde' nach der Methode von Dumas bestimmt.

1) $0,4515 \mathrm{Grm}$. lieferten $0,4533 \mathrm{Grm} . \mathrm{H}_{2} \mathrm{O}$ und $1,1490 \mathrm{Grm} . \mathrm{CO}_{2}$, entsprechend $11,16 \% \mathrm{H}$ und $69,40 \% \mathrm{C}$.

2) 0,2980 Grm. verbrannt lieferten 0,3055 Grm. $\mathrm{H}_{2} \mathrm{O}$ und 0,7575 Grm. $\mathrm{CO}_{2}$, entsprechend $11,39 \% \mathrm{H}$ und $69,33 \% \mathrm{C}$.

3) $0,2380 \mathrm{Grm}$. lieferten $0,2570 \mathrm{Grm} . \mathrm{H}_{2} \mathrm{O}$ und 0,6090 Grm. $\mathrm{CO}_{2}$, entsprechend $11,99 \% \mathrm{H}$ und $69,78 \% \mathrm{C}$. 
320 Parcus: Ueber einige neue Gehirnstoffe.

4) 0,4164 Grm. lieferten $7,9 \mathrm{Ccm}$. bei $12^{\circ}$ und $760 \mathrm{Mm}$. = 0,00937807 Grm. $\mathrm{N}=2,25 \% \mathrm{~N}$ bei $0^{\circ}$ und $760 \mathrm{Mm}$.

5) $0,4390 \mathrm{Grm}$. lieferten $7,4 \mathrm{Ccm}$. bei $5^{0}$ und $752 \mathrm{Mm} .=0,0089568$ Grm. $=2,04 \% \mathrm{~N}$ bei $0^{\circ}$ und $760 \mathrm{Mm}$.

2 mal umkrystallisirtes Cerebrin.

$\begin{array}{rrrrc} & 1 . & 2 . & 3 . & \text { Mittel. } \\ \mathrm{C}= & 69,40 & 69,33 & 69,78 & 69,50 \\ \mathrm{H}= & 11,16 & 11,39 & 11,99 & 11,51 \\ \mathrm{~N}= & 2,25 & 2,04 & - & 2,145\end{array}$

Durch abermaliges Umkrystallisiren auf die oben angegebene Weise erhielt ich weitere Mengen der Substanzen $M$ und $\mathrm{D}$.

Das jetzt fünfmal umkrystallisirte Cerebrin ergab bei der Analyse folgende Zahlen:

1) $0,3445 \mathrm{Grm}$. lieferten $0,3502 \mathrm{Grm} . \mathrm{H}_{2} \mathrm{O}$ and $0,8750 \mathrm{Grm} . \mathrm{CO}_{2}$, entsprechend $11,29 \% \mathrm{H}$ und $69,27 \% \mathrm{C}$.

2) $0,3097 \mathrm{Grm}$. lieferten $0,3128 \mathrm{Grm} . \mathrm{H}_{2} \mathrm{O}$ und $0,7909 \mathrm{Grm} . \mathrm{CO}_{2}$, entsprechend $11,22 \% \mathrm{H}$ und $69,64 \% \mathrm{C}$.

3) $0,2470 \mathrm{Grm}$. lieferten $0,2535 \mathrm{Grm} . \mathrm{H}_{2} \mathrm{O}$ und 0,6280 Grm. $\mathrm{CO}_{2}$, entsprechend $11,40 \% \mathrm{H}$ and $69,34 \% \mathrm{C}$.

4) $0,5970 \mathrm{Grm}$. lieferten $10,8 \mathrm{Ccm}$. $\mathrm{N}$ bei $13,5^{\circ}$ und $767,5 \mathrm{Mm} .=$ $0,0128629 \mathrm{Grm} .=2,15 \% \mathrm{~N}$ bei $0^{\circ}$ und $760 \mathrm{Mm}$.

5) $0,7765 \mathrm{Grm}$. lieferten $14,4 \mathrm{Ccm}$. $\mathrm{N}$ bei $18^{\circ}$ und $765,5 \mathrm{Mm}$. $=$

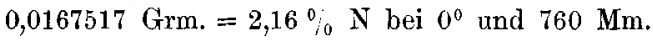

$$
\begin{array}{rrrrc} 
& \multicolumn{5}{c}{5 \text { mal umkrystallisirtes }} & \text { Cerebrin. } & \\
& 1 . & 2 . & 3 . & \text { Mittel. } \\
\mathrm{C}= & 69,27 & 69,34 & 69,64 & 69,42 \\
\mathrm{H}= & \mathbf{1 1 , 2 9} & \mathbf{1 1 , 4 0} & \mathbf{1 1 , 2 2} & \mathbf{1 1 , 3 0} \\
\mathrm{N}= & \mathbf{2 , 1 5} & \mathbf{2 , 1 6} & - & \mathbf{2 , 1 5 5}
\end{array}
$$

Die Mittel der Analysen von zweimal und fünfmal umkrystallisirtem Cerebrin ergaben demnach:

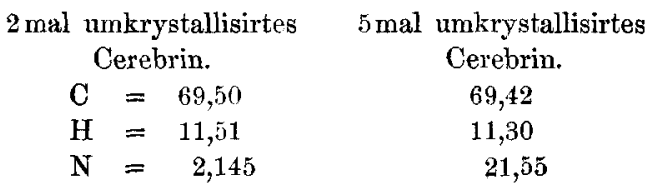

woraus eine genügende Uebereinstimmung hervorgeht. Dieses Resultat bewies, dass die Zusammensetzung des Cerebrins durch weiteres Umkrystallisiren nicht mehr verändert wird, und berechtigte zu dem Schluss, dass die in der Mutterlauge 
zurückgebliebene Substanz entweder Cerebrin oder ein dem Cerebrin nahe stehender, vielleicht isomerer Körper sei.

Um dies mit Sicherheit festzustellen, wurde das Cerebrin wieder mehrmals umkrystallisirt. Die Ausscheidungen in der Mutterlauge verminderten sich mit jeder Krystallisation, und hörten schliesslich bis auf geringe Mengen eines pulverigen Niederschlages, welcher dem gelöst gebliebenen Cerebrin zugeschrieben werden konnte, ganz auf. Dieser Umstand bestätigte, dass $M$ kein Cerebrin sei, denn letzteres hätte sich jedes Mal in gleichen Mengen aus der Mutterlauge ausscheiden müssen.

Im Ganzen war nun 8 Mal umkrystallisirt worden. Weitere Analysen ergaben folgende Resultate:

1) $0,0846 \mathrm{Grm}$. lieferten verbrannt $0,0865 \mathrm{Grm} . \mathrm{H}_{2} \mathrm{O}$ and 0,2150 Grm. $\mathrm{CO}_{2}$, entsprechend $11,36 \% \mathrm{H}$ und $69,31 \% \mathrm{C}$.

2) $0,3043 \mathrm{Grm}$. lieferten verbrannt $5,4 \mathrm{Ccm}$. $\mathrm{N}$ bei $16^{\circ}$ und 749,5 $\mathrm{Mm} .=0,00620733 \mathrm{Grm} . \mathrm{N}=2,04 \% \mathrm{~N}$ bei $0^{\circ}$ und $760 \mathrm{Mm}$.

Folgende Tabelle enthält die Mittel der Analysen des $2 \mathrm{mal}$, $5 \mathrm{mal}$ und $8 \mathrm{mal}$ umkrystallisirten Cerebrins.

$\begin{array}{ccc}2 \text { mal umkryst. } & 5 \text { mal } 1: \text { mkryst. } & 8 \text { mal umkryst. } \\ \text { Cerebrin. } & \text { Cerebrin. } & \text { Cerebrin. } \\ \mathrm{C}=69,50 & 69,42 & 69,31 \\ \mathrm{H}=11,51 & 11,30 & 11,36 \\ \mathrm{~N}=2,145 & 2,155 & 2,04\end{array}$

Leider berechtigten die gewomenen Zahlen nicht zur Aufstellung einer Formel, da Cerebrin, im Platintiegel verbrannt, eine nicht unbedeutende Menge Asche hinterliess.

$0,6100 \mathrm{Grm}$. lieferten $0,0018 \mathrm{Grm} .=0,295 \%$ Asche.

In demselben konnte Kohlensäure und auch Barium nachgewiesen werden, was für die Gegenwart einer fetten Säure sprach. Zur Entfernung dieser Verunreinigungen wurde Cerebrin mit Wasser angerieben, in den erhaltenen Brei Kohlensäure eingeleitet und dann abfiltrirt.

0,5572 Grm. lieferten nun 0,0007 Grm. $=0,12 \%$ Asehe.

Durch wiederholtes Waschen mit kohlensäurehaltigem Wasser wurde alsdann ein Cerebrin erhalten, welches, aus Alkohol umkrystallisirt und verbrannt, nur noch unwägbare Journal f. prakt. Chemie [2] Bd. 24. 


\section{Parcus: Ueber einige neue Gehirnstoffe.}

Spuren von Asche hinterliess. Eine Analyse des Cerebrins gab folgendes Resultat:

$0,1773 \mathrm{Grm}$. lieferten verbrannt $0,1853 \mathrm{Grm}$. $\mathrm{H}_{2} \mathrm{O}$ und $0,4475 \mathrm{Grm}$. $\mathrm{CO}_{2}$, entsprechend $11,61 \% \mathrm{H}$ und $68,84 \%_{0} \mathrm{C}$.

Das so gereinigte Cerebrin fiel aus alkoholischen Lösungen als weisses Pulver zu Boden, mikroskopische Kugeln darstellend. In langsam verdunstenden Lösungen wurden häufig am Rande des Uhrglases in ausserordentlich geringen Mengen auftretende feine Blättchen beobachtet. Mehrfaches Umkrystallisiren aus Alkohol, in welchem diese löslicher schienen als Cerebrin, genügte nicht, sie zu entfernen, so dass die Annahme nahe lag, Cerebrin möge durch Kochen mit diesem Lösungsmittel eine Zersetzung erleiden. Fünfbis sechstägiges Kochen mit Alkohol am Rückftusskühler, oder ebeniso langes Erhitzen mit dieser Flüssigkeit bis 100" im zugeschmolzenen Rohre reichten jedoch nicht hin, das Cerebrin zu verändern. Die Reinigungsversuche wurden deshalb wieder aufgenommen, und es gelang schliesslich, durch fortgesetztes Umkrystallisiren Cerebrin zu erhalten, welches keine Spuren mehr von jenen Blättchen aufwies. Somit blieb nur der Schluss übrig, dass dieselben aus der alkoholischen Lösung durch das ausfallende Cerebrin stets mit niedergerissen worden waren.

Der Stickstoffgehalt der Substanz M war folgender:

$0,5954 \mathrm{Grm}$. lieferten verbrannt $11,00 \mathrm{Ccm}$. $\mathrm{N}$ bei $14^{\circ}$ und 746 $\mathrm{Mm}$. Druck $=0,01269971 \mathrm{Grm} .=2,13 \%_{0} \mathrm{~N}$ bei $0^{\circ}$ und $760 \mathrm{Mm}$.,

also eben so viel wie die Analysen des Cerebrins selbst ergeben hatten. M konnte aber noch Cerebrin enthalten. Eine Probe wurde in absolutem Alkohol gelöst, und ein Theil der Lösung auf dem Uhrglase verdunstet. Es schieden sich Cerebrinkugehn aus, umhüllt von netzartig verzweigten Fäden. Die von diesen Kugeln abgegossene Lösung lieferte bei weiterer Verdunstung sehr feine, rosettenförmig gruppirte Nadeln und zugleich am Rande in geringer Menge Blättchen denen gleich, welche sich schon früher als Verunreinigungen des Cerebrins ergeben hatten. Demnach bestand $M$ aus einem Gemenge, von drei Substanzen. Zweien von diesen 
gebe ich, als bis jetzt noch nicht beobachteten, unbekannten Körpern die Namen Homocerebrin und Enkephalin.

Von Cerebrin wurden sie auf folgende Weise getrennt:

Eine heisse concentrirte Lösung von $M$ wurde langsam abgekühlt und einige Zeit bei der Temperatur erhalten, bei welcher Ausscheidung beginnt. Dabei fiel das Cerebrin als der unlöslichere Theil zu Boden; aus zu concentrirten Lösungen scheidet sich aber auch ein Theil des in Nadeln krystallisirenden Homocerebrins aus. Das Filtrat enthielt Homocerebrin, durch Enkephalin verunreinigt. Aus der Lösung schied sich das Homocerebrin als Gallerte oder in Hänten an den Wänden haftend aus. Daneben fanden sich auch $\mathrm{Ku}$ geln, welche sich als ein Gemenge von Homocerebrin und Enkephalin ergaben.

Da die Untersuchung von $M$ drei Substanzen geliefert hatte, war es wahrscheinlich, dass auch D ein solches Gemenge darstellte. Die fein gepulverte Substanz wurde mit kaltem Aether behandelt, welcher aber nichts aufnahm. Dann wurde der Stickstoffgehalt bestimint. D lieferte:

$$
\begin{aligned}
& 2,70{ }^{\circ} \mathrm{N} \\
& 2,36 " \Rightarrow \\
& 2,51 " \Rightarrow "
\end{aligned}
$$

Dieses giebt als Mittel 2,53\% $\%_{0} \mathrm{~N}$, also fast $0,5 \%_{0} \mathrm{~N}$ mehr, als Cerebrin enthält.

Die mikroskopische Untersuchung ergab sodann, dass D aus Homocerebrin und Enkephalin bestand.

Der in D gefundene höhere Stickstoffgehalt musste dem Enkephalin zukommen, denn die Analysen des Cerebrins sowohl wie diejenigen von $\mathrm{M}$, welches letzteres der Hauptsache nach ein Gemenge von Cerebrin und Homocerebrin dar. stellte, hatten weniger Stickstoff ergeben. Da aus der Untersuchung hervorging, dass Homocerebrin von Enkephalin in alkoholischer Lösung sich nicht trennen liess, wurde zur Scheidung beider Aceton benutzt, und zunächst durch häufiges Umkrystallisiren das aus M gewonnene Homocerebrin vollständig von Enkephalin befreit.

Will man sich von der Reinheit der Produkte überzeugen, so nimmt man eine ganz geringe Menge der zu prü- 
324 Parcus: Ueber einige neue Gehirnstoffe.

fenden Substanz in heissem Alkohol auf, und zwar nur so viel, dass dieselbe auch nach clem Erkalten gelöst bleibt. Man lässt dann einen Theil der Lösung langsam, aber nicht vollständig verdunsten und untersucht den Rückstand unter dem Mikroskop.

Bei rascher Ausscheidung fallen sowohl Homocerebrin, als auch Enkephalin, insbesondere aber ein Gemenge von beiden, in mikroskopischen Kugeln nieder, welche sich nur wenig von denen des Cerebrins unterscheiden. Solche Produkte können alsdann zu dem falschen Schlusse verleiten, Homocerebrin and Enkephalin seien fähig, unter geeigneten Verhältnissen in Cerebrin überzugehen.

Bei vollständigem Verdunsten der Lösung schrumpfen alle drei Körper zu auscheinend structurlosen Massen zusammen.

Das gereinigte Cerebrin wurde nun analysirt. Zu den Analysen 3) und 4) wurde solches Cerelorin verwendet, welches aus der Mutterlauge von umkrystallisirtem Cerebrin erhalten worden war.

1) $0,3786 \mathrm{Grm}$. lieferten $0,3900 \mathrm{Grm}$. $\mathrm{H}_{2} \mathrm{O}$ und $0,9596 \mathrm{Grm}$. $\mathrm{CO}_{2}$, entsprechend $11,45^{\circ}{ }_{10} \mathrm{H}$ und $69,125{ }^{\circ}{ }_{0} \mathrm{C}$.

2) $0,2507 \mathrm{Grm}$. lieferten $0,2608 \mathrm{Grm} . \mathrm{H}_{2} \mathrm{O}$ und 0,6344 Grm. $\mathrm{CO}_{2}$, entsprechend $11,55 \% \mathrm{H}$ und $69,01 \% \mathrm{C}$.

3) $0,3426 \mathrm{Grm}$. lieferten 0,3535 Grm. $\mathrm{H}_{2} \mathrm{O}$ und $0,8689 \mathrm{Grm} . \mathrm{CO}_{2}$, entsprechend $11,465 \% \mathrm{H}$ und $69,17 \% \mathrm{C}$.

4) $0,3423 \mathrm{Grm}$. lieferten $0,3510 \mathrm{Grm}$. $\mathrm{H}_{2} \mathrm{O}$ und $0,8670 \mathrm{Grm} . \mathrm{CO}$, entsprechend $11,40 \% \mathrm{H}$ und $69,07 \% \mathrm{C}$.

5) 0,157 Grm. lieferten 0,163 Grm. H.O und 0,3982 Grm. CO, entsprechend $11,54 \%_{0} \mathrm{H}$ and $69,17 \%_{0} \mathrm{C}$.

6) 0,2306 Grm. lieferten 0,237 Grm. H,O und 0,5830 Grm. CO, entsprechend $11,42 \% \mathrm{H}$ und $68,95 \% \mathrm{C}$.

7) 0,5065 Grm. lieferten $9 \mathrm{Cem}$. bei $19^{\circ}$ und $761 \mathrm{Mm},=0,010364$ Grm. $\mathrm{N}=2,04^{\circ}{ }_{0} \mathrm{~N}$ bei $0^{\circ}$ und $760 \mathrm{Mm}$.

8) $0,3148 \mathrm{Grm}$. lieferten 6,4 Ccm. bei $19^{\circ}$ und $756 \mathrm{Mm}$.= $0,00732081 \mathrm{Grm} . \mathbf{N}=2,33 \% \mathrm{~N}$ bei $0^{\circ}$ und $760 \mathrm{Mm}$.

9) $0,4300 \mathrm{Grm}$. lieferten $7,5 \mathrm{Ccm}$. bei $16^{\circ}$ und $755 \mathrm{Mm}$. $=0,008692$ Grm. $\mathbf{N}=2,02 \% \mathrm{~N}$ bei $0^{\circ}$ und $760 \mathrm{Mm}$.

10) $0,4525 \mathrm{Grm}$. lieferten $8,5 \mathrm{Ccm}$. bei $20^{\circ}$ und $751 \mathrm{Mm}$. $=$ 0,00960594 Grm. $\mathrm{N}=2,12 \% \mathrm{~N}$ bei $0^{\circ}$ und $760 \mathrm{Mm}$.

Folgende Tabelle enthält die Resultate dieser Analysen zusammengestellt: 
Parcus: Ueber einige nene Gehirnstoffe. 325

$\begin{array}{rccccccr}\mathrm{C}= & 1 . & 2 . & .3 . & 4 . & 5 . & 6 . & \text { Mittel. } \\ \mathrm{H}= & \mathbf{2} 9,125 & 69,01 & 69,17 & 69,07 & 69,17 & 68,95 & 69,08 \\ \mathrm{~N}= & \mathbf{1 1 , 4 5} & 11,55 & \mathbf{1 1 , 4 6 5} & 11,40 & 11,54 & 11,42 & 11,47 \\ 2,04 & \mathbf{2 , 0 2} & 2,12 & 2,33 & - & - & \mathbf{2 , 1 3}\end{array}$

Vermittelst dieser Zahlen lassen sich folgende Formeln aufstellen:

$$
\begin{aligned}
& \text { 1) } \mathrm{C}_{70} \mathrm{H}_{140} \mathrm{~N}_{2} \mathrm{O}_{13} \\
& \text { Berechnet. Gefunden. } \\
& \mathrm{C}=69,08 \% \quad 69,08 \% \\
& \mathrm{H}=11,51, \quad 11,47, \\
& \mathrm{~N}=2,30, \quad 2,13, \\
& \mathrm{O}=17,11, \quad 17,32, \\
& \text { 2) } \mathrm{C}_{76} \mathrm{H}_{154} \mathrm{~N}_{2} \mathrm{O}_{14} \\
& \mathrm{C}=69,19 \% \\
& \mathrm{H}=11,68, " \quad 11,47, \\
& \mathbf{N}=2,12, \quad 2,13, \\
& \mathrm{O}=17,01, \quad 17,32 \text {, } \\
& \text { 3) } \mathrm{C}_{80} \mathrm{H}_{160} \mathrm{~N}_{2} \mathrm{O}_{15} \\
& \mathrm{C}=69,16 \% \quad 69,08 \% \\
& \mathrm{H}=11,53, \quad 11,47, \\
& \mathrm{~N}=2,02 n \quad 2,13 " \\
& 0=17,29,17,32,
\end{aligned}
$$

Letztere Formel dürfte der Zusammensetzung des Cerebrins am nächsten kommen, denn die dafür berechneten Mengen stimmen mit den Zahlen, welche die Analysen ergaben, insbesondere aber mit den gefundenen Stickstoffmengen, gut überein, mit Ausnahme der einen Analyse, welche $2,33 \% \mathrm{~N}$ lieferte.

Dann folgen die Analysen von Homocerebrin:

1) $0,1494 \mathrm{Grm}$. lieferten $0,1603 \mathrm{Grm} . \mathrm{H}_{2} \mathrm{O}$ und $0,3842 \mathrm{Grm}$. $\mathrm{CO}_{2}$, entsprechend $11,92 \% \mathrm{H}$ und $70,135 \% \mathrm{C}$.

2) $0,145 \mathrm{Grm}$. lieferten $0,1514 \mathrm{Grm} . \mathrm{H}_{2} \mathrm{O}$ und $0,3735 \mathrm{Grm} . \mathrm{CO}_{2}$, entsprechend $11,60 \% \mathrm{H}$ und $70,25 \% \mathrm{C}$.

3) 0,316 Grm. lieferten 0,329 Grm. $\mathrm{H}_{22} \mathrm{O}$ und 0,812 Grm. $\mathrm{CO}_{2}$, entsprechend $11,57 \% \mathrm{H}$ und $70,08 \% \mathrm{C}$.

4) $0,2451 \mathrm{Grm}$. lieferten $0,255 \mathrm{Grm} . \mathrm{H}_{2} \mathrm{O}$ und 0,6283 Grm. $\mathrm{CO}_{2}$, entsprechend $11,56 \% \mathrm{H}$ und $69,91 \% \mathrm{C}$.

5) $0,163 \mathrm{Grm}$. lieferten $0,1684 \mathrm{Grm} . \mathrm{H}_{2} \mathrm{O}$ und $0,4714 \mathrm{Grm} . \mathrm{CO}_{2}$, entsprechend $11,48 \% \mathrm{H}$ und $69,84 \% \mathrm{C}$. 
326 Parcus: Ueber einige neue Gehirnstoffe.

6) $0,2705 \mathrm{Grm}$. lieferten $0,2785 \mathrm{Grm}$. $\mathrm{H}_{2} \mathrm{O}$ und $0,694 \mathrm{Grm} . \mathrm{CO}_{2}$, entsprechend $11,44 \%_{0} \mathrm{H}$ und $69,97 \%_{\%_{0}} \mathrm{C}$.

7) $0,5341 \mathrm{Grm}$. lieferten $9,9 \mathrm{Ccm}$. bei $18^{\circ}$ und $765,5 \mathrm{Mm}$. = 0,01138042 Grm. $=2,13{ }^{\circ}{ }_{0} \mathrm{~N}$ bei $0^{\circ}$ nnd $760 \mathrm{Mm}$.

3) $0,3260 \mathrm{Grm}$. lieferten $6,5 \mathrm{Ccm}$. bei $16^{\circ}$ und $749 \mathrm{Mm}$. = 0,0074663 Grm. $=2,29 \% \mathrm{~N}$ bei $0^{0}$ und $760 \mathrm{Mm}$.

9) $0,397 \mathrm{Grm}$. lieferten $7,9 \mathrm{Ccm}$. bei $19^{\circ}$ und $756 \mathrm{Mm} .=0,0090366$ Grm. $=2,27 \% \mathrm{~N}$ bei $0^{0}$ und $760 \mathrm{Mm}$.

Resultate dieser Analysen:

\begin{tabular}{|c|c|c|c|c|c|c|c|c|}
\hline C & $=$ & $\begin{array}{c}1 . \\
70,135\end{array}$ & $\begin{array}{c}2 . \\
70,25\end{array}$ & $\begin{array}{c}3 . \\
69,01\end{array}$ & $\begin{array}{c}4 . \\
70,08\end{array}$ & $\begin{array}{c}5 . \\
69,84\end{array}$ & $\begin{array}{c}6 . \\
69,97\end{array}$ & $\begin{array}{c}\text { Mittel. } \\
70,06\end{array}$ \\
\hline$H$ & $=$ & 11,92 & 11,60 & 11,56 & 11,57 & 11,48 & 11,44 & 11,595 \\
\hline $\mathrm{N}$ & $=$ & 2,13 & 2,29 & 2,27 & - & - & - & 2,23 \\
\hline
\end{tabular}

Formeln für Homocerebrin:

1) $\mathrm{C}_{70} \mathrm{H}_{132} \mathrm{~N}_{2} \mathrm{O}_{12}$

\begin{tabular}{|c|c|c|}
\hline & Berechnet. & Gefunden. \\
\hline $\mathrm{C}$ & $=70,12 \%$ & $70,06 \%$ \\
\hline $\mathrm{H}$ & $=11,52 n$ & 11,595 \\
\hline $\mathbf{N}$ & 2,33, & 2,23 \\
\hline O & $=16,03$, & $16,115 \%$ \\
\hline \multicolumn{3}{|c|}{ 2) $\mathrm{C}_{76} \mathrm{H}_{152} \mathrm{~N}_{2} \mathrm{O}_{13}$} \\
\hline $\mathrm{C}$ & $=70,155^{0}$ & $70,06 \%$ \\
\hline $\mathrm{H}$ & $11,69 \quad$, & 11,595 \\
\hline $\mathbf{N}$ & 2,155, & $2,23 \quad$, \\
\hline $\mathrm{O}$ & $=16,00 \quad$, & $16,115 n$ \\
\hline \multicolumn{3}{|c|}{ 3) $\mathrm{O}_{40} \mathrm{H}_{158} \mathrm{~N}_{2} \mathrm{O}_{14}$} \\
\hline $\mathrm{C}$ & $=70,070_{0}^{0}$ & $70,(16 \%$ \\
\hline $\mathrm{H}$ & 11,53, & 11,595, \\
\hline $\mathbf{N}$ & 2,04, & 2,23 \\
\hline 0 & $=16,35$, & 16,115, \\
\hline
\end{tabular}

Man ersieht aus dieser Reihe von Formeln, welche sich für Cerebrin und Homocerebrin aufstellen lassen, dass vorläufig ohne genauere Kenntniss der Spaltungsprodukte die atomistische Zusammensetzung von Cerebrin und Homocerebrin mit Sicherheit nicht angegeben werden kann. Doch lassen sich zwischen den Formeln von Cerebrin und Homocerebrin interessante Beziehungen finden. Jeder Formel für Cerebrin entspricht eine solche für Homocerebrin, welche sich nur durch ein Molekül Wasser unterscheidet. 
Parcus: Ueber einige nene Gehirnstoffe. 327

Cerebrin. Homocerebrin.

$$
\begin{aligned}
& \mathrm{C}_{70} \mathrm{H}_{140} \mathrm{~N}_{2} \mathrm{O}_{13}=\mathrm{C}_{70} \mathrm{H}_{138} \mathrm{~N}_{2} \mathrm{O}_{12}+\mathrm{H}_{2} \mathrm{O} \\
& \mathrm{C}_{76} \mathrm{H}_{154} \mathrm{~N}_{2} \mathrm{O}_{14}=\mathrm{C}_{76} \mathrm{H}_{152} \mathrm{~N}_{2} \mathrm{O}_{13}+\mathrm{H}_{2} \mathrm{O} \\
& \mathrm{C}_{30} \mathrm{H}_{160} \mathrm{~N}_{2} \mathrm{O}_{15}=\mathrm{C}_{30} \mathrm{H}_{158} \mathrm{~N}_{2} \mathrm{O}_{14}+\mathrm{H}_{2} \mathrm{O}
\end{aligned}
$$

Vergleicht man weiter Formel 1) für Cerebrin mit Formel 2) für Homocerebrin, so kommt man zu der Vermuthung, dass man es mit homologen Körpern zu thun habe.

$$
\frac{\mathrm{C}_{76} \mathrm{H}_{152} \mathrm{~N}_{2} \mathrm{O}_{13}}{\mathrm{C}_{70} \mathrm{H}_{140} \mathrm{~N}_{2} \mathrm{O}_{13}}=\left(\mathrm{CH}_{2}\right)_{6}
$$

Dasselbe Resultat liefert Formel 2) für Cerebrin, verglichen mit der Formel 1) für Homocerebrin:

$$
\begin{aligned}
& \mathrm{C}_{76} \mathrm{H}_{154} \mathrm{~N}_{2} \mathrm{O}_{14} \\
& \mathrm{C}_{70} \mathrm{H}_{138} \mathrm{~N}_{2} \mathrm{O}_{12} \\
& \mathrm{C}_{6} \mathrm{H}_{16} \quad \mathrm{O}_{2}=\left(\mathrm{CH}_{2}\right)_{6}+2 \mathrm{H}_{2} \mathrm{O}
\end{aligned}
$$

Von Enkephalin habe ich nur wenige Analysen ausgeführt, da zu weiteren das Material nicht ausreichte.

1) $0,1425 \mathrm{Grm}$. lieferten $0,151 \mathrm{Grm} . \mathrm{H}_{2} \mathrm{O}$ und $0,358 \mathrm{Grm}$. $\mathrm{CO}_{2}$, entsprechend $11,77 \% \mathrm{H}$ und $68,52 \% \mathrm{C}$.

2) $0,2175 \mathrm{Grm}$. lieferten $0,224 \mathrm{Grm} . \mathrm{H}_{2} \mathrm{O}$ und $0,5445 \mathrm{Grm} . \mathrm{CO}_{2}$, entsprechend $11,44 \% \mathrm{H}$ und $68,28 \% \mathrm{C}$.

3) $0,217 \mathrm{Grm}$. lieferten $5,8 \mathrm{Ccm}$. bei $19^{\circ}$ und $755,5 \mathrm{Mm} .=0,0067151$ Grm. $=3,09 \% \mathrm{~N}$ bei $0^{\circ}$ und $760 \mathrm{Mm}$.

Resultate der Analysen von Enkephalin.

$$
\begin{array}{rrrr} 
& \mathbf{1 .} & 2 . & \text { Mittel. } \\
\mathrm{C}= & 68,52 & 68,28 & 68,40 \\
\mathrm{H}= & 11,77 & \mathbf{1 1 , 4 4} & 11,60 \\
\mathrm{~N}= & \mathbf{3}, 09 & - & \mathbf{3}, 09
\end{array}
$$

Für Enkephalin lässt sich die Formel $\mathrm{C}_{102} \mathrm{H}_{206} \mathrm{~N}_{4} \mathrm{O}_{19}$ aufstellen.

$$
\begin{aligned}
& \text { Berechnet. Gefunden. } \\
& \mathrm{C}=68,38 \% \quad 68,40 \% \\
& \mathrm{H}=11,51, \quad 11,60, \\
& \mathrm{~N}=3,13, \quad 3,09, \\
& \mathrm{O}=16,98, \quad 16,91 \%
\end{aligned}
$$




\section{Parcus: Ueber einige neue Gehirnstoffe.}

Zwei Stickstoffbestimmungen wurden mit anderer Substanz ausgeführt, welche jedoch, wie es scheint, nicht die gleiche Reinheit besass.

1) $0,3615 \mathrm{Grm}$. lieferten $7,7 \mathrm{Ccm}$. bei $13^{\circ}$ und $757 \mathrm{Mm} .=2,57^{\circ}, 0$ $\mathrm{N}$ bei $0^{\circ}$ und $760 \mathrm{Mm}$.

2) 0,3655 Grm. lieferten $7,9 \mathrm{Ccm}$. bei $14^{\circ}$ und $755 \mathrm{Mra} .=2,55 \%$ $\Lambda^{\top}$ bei $0^{\circ}$ und $760 \mathrm{Mm}$.

Hieraus ergeben sich $0,50 \% \mathrm{~N}$ weniger, als aus der ersten Bestimmung.

\section{Cerebrin.}

Das gereinigte Cerebrin stellt getrocknet einen schneeweissen Körper dar, welcher von kochendem Alkohol leicht aufgenommen wird und beim Erkalten der Lösung sich als krystallinisch erscheinendes Pulver wieder ausscheidet. In der Wärme löst es sich in Aceton, Chloroform, Benzol, Eisessig und vielen anderen Mitteln auf, ist dagegen in kaltem, wie auch in heissem Aether gänzlich unlöslich. Aus mehreren Lösungsmitteln, insbesondere aber aus Aceton fällt es nicht als Pulver, sondern in Flocken nieder, welche unter dem Mikroskop zum Theil verfilzt, zum Theil als Kugeln erscheinen. Das aus alkoholischer Lösung gewonnene Cerebrin besitzt dagegen stets das Aussehen von farblosen, durchsichtigen Globuliten, welche sich zu traubenförmigen Gebilden von ausserordentlicher Zartheit und Schönheit der Gestaltung ordnen. Die einzelnen Kügelchen sind dabei zum Theil so klein, dass sie selbst bei 900 facher Vergrösserung nur als feine Lichtpunkte erscheinen. Sie erweisen sich dann als sehr schwach anisotrop. In ausgebildeten Krystallen habe ich es niemals erhalten. Reibt man es mit conc. Schwefelsäure an, so erhält man eine hellgelbe klare Flüssigkeit, welche bei längerem Stehen an der Luft eine purpurrothe, später grau werdende Haut abscheidet, wobei die Lösung farblos wird. Das im Vacuum über Schwefelsäure getrocknete Cerebrin vermag aus mit Feuchtigkeit gesättigter Luft etwas über $2 \%$ Wasser aufzunehmen, ist also nicht stark hygroskopisch. 
Im Reagensglas sehr vorsichtig erhitzt, schmilzt es zu einer klaren, farblosen Flüssigkeit ohne Zersetzung. Erhitzt man es dagegen behufs Schmelzpunktbestimmung, so tritt bei $145^{\circ}$ eine Zersetzung ein, welche sich durch schwache Gelbfärbung kundgiebt; man kann alsdann bis auf $160^{\circ} \mathrm{er}$ hitzen, ohne dass die Färbung erheblich stärker wird. Ueber $160^{\circ}$ tritt starke Bräunung ein, verbunden mit beginnender Schmelzung; anhaltend auf $170^{\circ}$ erhitzt, verwandelt es sich in eine braune Flüssigkeit, welche beim Erkalten zu einer zähen Masse erstarrt. Nimmt man diese mit Alkohol auf und verdunstet die erhaltene Lösung, so scheiden sich weisse Flocken ab, welche aus mikroskopisch kleinen Kugeln bestehen und kein Cerebrin mehr sind. Wird die geschmolzene Masse im Reagensglas weiter erhitzt, so entwickelt sich zunächst ein nicht unangenehmer Geruch nach gebratenem Fleisch, dann aber entweichen scharfe, beissende Dämpfe, wie sie Talg oder andere Fette liefern, wenn man sie mit saurem schwefelsauren Kali erhitzt. Der Rückstand verbrennt auf dem Platinblech unter Entflammung und hinterlässt eine Kohle, welche beim gelinden Glühen rasch und vollständig verschwindet. In heissem Wasser quillt Cerebrin auf, jedoch nicht stark. Küblt man ab, so setzt es sich in Flocken zu Boden.

Verbindungen von Cerebrin mit Basen, Salzen oder Säuren wurden trotz zahlreicher, in dieser Richtung angestellter Versuche nicht erhalten. In seinen Lösungen reagirte es neutral.

Durch anhaltendes Kochen mit Wasser erleidet es keine Zersetzung. Eben so wenig wird es durch einmaliges Aufkochen mit concentrirtem Barytwasser angegriffen, dagegen vollständig zersetzt dureh fortgesetztes Kochen mit dieser Base.

Die geringen Veränderungen, welche es nach mehrstündigem Kochen am Rückflusskühler mit alkoholischer Kalilauge zeigte, beweisen seine ausserordentliche Widerstandsfähigkeit gegen die Einwirkung dieses Reagenses.

Die Produkte der Zersetzung des Cerebrins, welche man durch Einwirkung von Salzsäure oder mehrstündiges Kochen 
330 Parcus: Ueber einige neue Gehirnstoffe.

mit Barythydrat erhält, habe ich zu studiren unternommen, ohne aber zu wesentlichen Resultaten zu gelangen.

Einmal verhalten sich jene Zersetzungsprodukte den Lösungsmitteln gegenüber ziemlich ähnlich, dann aber scheint es, dass die primär gebildeten Produkte durch die angewandten Reagentien sofort eine weitere Veränderung erleiden, welche ein unentwirrbares Gemenge zur Folge hat. Die Resultate dieser Versuche führe ich, so weit sie erwähnenswerth sind, hier an.

Wird Cerebrin mit verdünnter Salzsäure mehrere Stunden gekocht, so schwimmen schliesslich nur noch einige weisse Flocken in der sonst gelblich gefärbten Flüssigkeit.

Das salzsaure Filtrat, welches mit Leichtigkeit alkalische Kupferlösung reducirt, scheint ausser anderen Produkten etwas Zucker zu enthalten, denn beim Eindampfen bilden sich stets humusartige Massen und der charakteristische Geruch nach verbranntem Zucker tritt auf. Bessere Resultate als die Behandlung mit Baryt und Salzsäure scheint die trockne Destillation des Cerebrins zu ergeben.

Aus Mangel an Material konnte jedoch ein eingehendes Studium der hierbei auftretenden Produkte nicht vorgenommen werden.

Das durch Erhitzen von Cerebrin erhaltene Destillat riecht nach verbranntem Zucker. Durch gleichzeitiges Behandeln desselben mit Aether und $\mathrm{W}_{\text {asser }}$ wird es gelöst. Die wässrige Lösung reagirt sauer und reducirt alkalisches Kupferoxyd, wie auch salpetersaures Silberoxyd. Die ätherische Lösung reagirt neutral und setzt beim Verdunsten eine in farblosen Blättchen krystallisirende Substanz $a b$, welche keinen Stickstoff enthält. Sie kann durch Umkrystallisiren aus Alkohol gereinigt werden und schmilzt dann bei $55^{\circ}$.

Fasst man die erhaltenen Resultate zusammen, so darf man aus der Reduction von alkalischer Kupferlösung, aus dem Geruch nach verbranntem Zucker, sowie der Bildung humusartiger Massen beim Eindampfen der salzsauren Lösung, endlich aus den Produkten der trocknen Destillation von Cerebrin auf die Gegenwart eines Kohlehydrates im Cere- 
Parcus: Ueber einige neue Gehirnstoffe.

brin schliessen. - Festzustellen, welcher Art dies ist, muss weiteren Untersuchungen überlassen bleiben.

Von den im Vorhergehenden beschriebenen Eigenschaften des Cerebrins verdient, abgesehen von seiner procentigen Zusammensetzung, als abweichend von früheren Angaben hervorgehoben zu werden:

1) die Bildung einer hellgelben Lösung mit concentrirter Schwefelsäure; 2) die Unlöslichkeit in heissem Aether; 3) die geringe Quellung in kochendem Wasser; 4) die geringen hygroskopischen Eigenschaften; 5) die Fähigkeit ohne Zersetzung zu schmelzen; 6) sein hoher Zersetzungspunkt überhaupt, und endlich 7) die eingreifende Veränderung, welche es durch längeres Kochen mit Barytwasser erleidet.

Fremy's Cerebrinsäure, welche durch Extraction des Gehirns mit heissem Aether erhalten worden war, konnte deshalb kein Cerebrin sein.

Müller's Cerebrin muss wegen des hohen Stickstoffgehaltes von $4,6 \%$, sowie anderer Umstände wegen, als verunreinigtes Cerebrin betrachtet werden. Otto's Cerebrin und Köhler's Myelomargarin stehen als stickstofffreie Substanzen in keiner Beziehung zu Cerebrin, oder können doch nur Spaltungsprodukte desselben sein. Von Thudichum's phosphorfreien Substanzen, dem

$$
\begin{aligned}
& \text { Cerebrin }{ }_{\text {Stearoconot }} \mathrm{C}_{34} \mathrm{H}_{68} \mathrm{~N}_{2} \mathrm{O}_{8} \\
& \text { Phrenosin } \quad \mathrm{C}_{34} \mathrm{H}_{67} \mathrm{NO}_{8} \\
& \text { Kerasin } \quad \mathrm{C}_{46} \mathrm{H}_{91} \mathrm{NO}_{9} \text {, }
\end{aligned}
$$

habe ich die procentige Zusammensetzung berechnet und in folgender Tabelle angeführt.

In der letzten Spalte ist das Mittel meiner Analysen angegeben.

Cerebrin und

Stearoconot

Phrenosin

Kerasin

Cerebrin.

Thudichum's. Thudichum's. Thudiehum's.

$\begin{array}{rrrrr}\mathrm{C}=64,56 & 66,125 & 68,91 & 69,08 \\ \mathrm{H}=10,76 & 10,86 & 11,36 & \mathbf{1 1 , 4 7} \\ \mathrm{N}=4,43 & 2,27 & 1,74 & 2,13 \\ \mathrm{O}=20,25 & 20,745 & 17,97 & 17,32\end{array}$




\section{Pareus: Ueber einige neue Gehirnstoffe.}

Man ersieht daraus, dass nur Thudichum's Kerasin sich dem Cerebrin nähert, während die drei anderen Substanzen in ihrer Zusammensetzung von demselben bedeutend abweichen. Da auch Homocerebrin, sowie Enkephalin bezüglich ihrer Zusammensetzung mit keinem der Körper Thu dichun's identisch sein können, andere Körper aber in dem alkoholischen Auszuge des coagulirten Gehirns, auf welchen sich meine Untersuchungen beziehen, nicht vorhanden sind, so wird es walırscheinlich, dass Thudichum in diesen Körpern entweder zersetzte, oder doch stark verunreinigte Cerebrine beschrieben hat.

Auch Geoghegan's ${ }^{1}$ ) Cerebrin ist in seiner Zusammensetzung von dem von mir dargestellten verschieden.

\begin{tabular}{|c|c|c|}
\hline \multicolumn{2}{|c|}{ Geoghegan's Cerebrin. } & Cerebrin. \\
\hline $\mathrm{C}$ & $=68,74$ & 69,08 \\
\hline $\mathrm{H}$ & $=10,91$ & 11,47 \\
\hline $\mathrm{N}$ & 1,44 & 2,13 \\
\hline 0 & $=18,91$ & 17,32 \\
\hline
\end{tabular}

Dass Homocerebrin oder Enkephalin seinem Cerebrin beigemengt gewesen seien, ist nicht denkbar, denn durch beide musste Kohlenstoff-, Wasserstoff- und Stickstoffgehalt erhöht worden sein. ${ }^{2}$ )

1) Hoppe-Seyler's Zeitschr. 3, 332.

") Wahrscheinlicher ist, dass er das Gemenge dieser Substanzen zu lange mit Barytwasser gekocht hat, weshalb sein Cerebrin zum grössten Theil aus Zersetzungsprodukten dieser Stoffe bestand. Diese Annahme gewinnt an Realität, wenn man beachtet, dass er seine Untersuchung im Hoppe-Seyler'schen Laboratorium ausführte, und er sich deshalb auch wohl bei Darstellung seines Cerebrins genau an die Angaben des Hoppe-Seyler'schen Handbuchs der physiologischen Chemie gehalten hat. Darin heisst es aber: „Dam wird eine Stunde mit Barytwasser gekocht." Dass er kein reines Cerebrin hatte, geht aus einer Angabe von ihm hervor, wonach sein Cerebrin alle die Eigenschaften besitzt, wie sie in Hoppe-Seyler's Handbuch beschrieben sind. Dort steht: „Auf diese Weise dargestellt bildet das Cerebrin ein leichtes, weisses, sehr hygroskopisches Pulver, welches beim Erhitzen über $80^{\circ}$ gebräunt wird. 
Parcus: Ueber einige neue Gehirnstoffe. 333

II. Homocerebrin.

Homocerebrin ist in geringeren Mengen im Gehirn enthalten als Cerebrin, denn die Ausbeute beträgt nur etwa $1 / 4$ des gewonnenen Cerebrins. Mit letzterem hat es die Haupteigenschaften gemein. Es löst sich in allen jenen Mitteln, welche schon beim Cerebrin angegeben worden sind, ausserdem in heissem Aether; es quillt in heissem Wasser auf, ohne einen Kleister zu bilden; Kochen damit zersetzt es nicht. Wird es mit heisser Salzsäure behandelt, so erhält man eine alkalisches Kupferoxyd reducirende Lösung. Durch anhaltendes Kochen mit Barytwasser wird es zersetzt.

Es ist nicht hygroskopisch, reagirt in seinen Lösungen neutral und ist unfähig Verbindungen einzugehen; es scheidet sich aus langsam verdunstenden Lösungen in ausserordentlich feinen, nadelförmigen Gebilden aus. Im Reagensglase erhitzt, schmilzt es ohne Zersetzung zu einer klaren, farblosen Flüssigkeit. Langsam erhitzt wird es bei niederer Temperatur zersetzt, als Cerebrin; bei $130^{\circ}$ bemerkt man eine schwache Gelbfärbung, welche mit Erhöhung der Temperatur stärker wird; bei $155^{\circ}$ verwandelt es sich in einen braunen, klaren Syrup, welcher sich wie geschmolzenes Cerebrin verhält. Ausser seiner Zusammensetzung unterscheidet es sich von diesem noch durch seine grössere Löslichkeit, sowie durch die Fähigkeit, Gallerten zu bilden. In viel A]kohol bleibt es nach dem Erkalten gelöst, bei rascher Abkühlung scheidet es sich daraus in Flocken ab. Abfiltrirt und getrocknet stellt es nicht, wie Cerebrin, ein leichtes, lockeres Pulver, sondern eine wachsartige, schwer zerreibliche Masse dar, welche Alkohol zurückhält.

Folgende Versuche schienen mir geeignet, die grössere Löslichkeit des Homocerebrins in absolutem Alkohol gegenüber dem Cerebrin darzuthun.

\section{Versuch.}

7,5 Grm. Cerebrin und 7,5 Grm. Homocerebrin wurden in je $250 \mathrm{Ccm}$. absolutem Alkohol unter Erwärmen gelöst und dann langsam abgekühlt. Nach 16 Stunden hatten sich 
334 Parcus: Ueber einige neue Gehirnstoffe.

7,25 Grm. Cerebrin und 6,95 Grm. Homocerebrin ausgeschieden, $0,25 \mathrm{Grm}$. Cerebrin und. 0,55 Grm. Homocerebrim waren gelöst geblieben. Dieser Versuch musste mehrere Male wiederholt werden, weil das Homocerebrin derartig zu einer Gallerte erstarrte, dass ich das Kölbchen umdrehen konnte, ohne dass der Alkohol ausfloss.

\section{Versuch.}

$5 \mathrm{Grm}$. Uerebrin wurden in $500 \mathrm{Ccm}$. Alkohol, 5 Grm. Homocerebrin in der gleichen Menge Alkohol gelöst, und nach 16 stündigem Stehen je $100 \mathrm{Ccm}$. der alkoholischen Lösungen verdunstet. Diese lieferten 0,0295 Grm. Cerebrin und 0,1240 Grm. Homocerebrin, oder auf einen Theil Cerebrin kamen 2688 Thle. Alkohol, auf einen Theil Homocerebrin 639 Thle. Alkohol.

\section{Versuch wie 2.}

Nach 24 stündigem Stehen gaben je $100 \mathrm{Ccm}$. verdunstet 0,0235 Grm. Cerebrin und 0,108 Grm. Homocerebrin; oder ein Theil Cerebrin hatte 3374 Thle. Alkohol, ein Theil Homocerebrin 734 Thle. erfordert.

\section{Versuch.}

1 Grm. Cerebrin und 1 Grm. Homocerebrin wurden in je $500 \mathrm{Ccm}$. Alkohol gelöst und 16 Stunden stehen gelassen. Je $100 \mathrm{Ccm}$. verdunstet lieferten 0,0295 Grm. Cerebrin und 0,134 Grm. Homocerebrin, oder 1 Thl. Cerebrin bedurfte zur Lösung 2688 Thle. Alkohol und 1 Thl. Homocerebrin 592 Thle. Aus den auf diese Weise erhaltenen Lösungen von Cerebrin und Homocerebrin schieden sich nach einiger Zeit abermals weitere Mengen Substanz aus. Es wurden. um der wahren Löslichkeit etwas näher zu kommen, diese kalt gesättigten Flüssigkeitsmengen noch einen Tag stehen gelassen und dann je $100 \mathrm{Ccm}$. verdunstet. Diese lieferten 0,0160 Grm. Cerebrin und 0,076 Grm. Homocerebrin, oder einen Theil Cerebrin auf 4956 Thle. Alkohol; und einen Theil Homocerebrin auf 1043 Thle. Alkohol.

Nach abermals 8 Tagen gaben je $100 \mathrm{Ccm}$. verdunstet 
0,008 Grm. Cerebrin und 0,0441 Grm. Homocerebrin oder ein Theil Cerebrin war gelöst in 9912 Thln. Alkohol; ein Theil Homocerebrin in 1800 Thln. Alkohol.

Nach weiteren 8 Tagen enthielten $100 \mathrm{Ccm}$. jeder Lö. sung noch 0,0065 Grm. Cerebrin und 0,0410 Grm. Homocerebrin. Demnach kam ein Theil Cerebrin auf 12200 Thle. Alkohol, ein Theil Homocerebrin auf 1934 Thle. Alkohol.

Die letzten Versuche, in welchen kalt gesättigte Lösungen bei der gleichen Temperatur stehen gelassen worden waren, zeigen, dass Cerebrin und Homocerebrin fähig sind übersättigte Lösungen zu bilden; sie theilen dieses Verhalten mit der Salicylsäure, deren Löslichkeit in Wasser nach den Versuchen von $\mathrm{Ost}^{1}$ ), entsprechend der Concentration bedeutenden Schwankungen unterliegt.

Die Beziehungen, welche zwischen den auf Grund der Analysen aufgestellten Formeln von Cerebrin und Homocerebrin gefunden wurden, waren zweierlei. Einmal liess sich Cerebrin als Hydrat von Homocerebrin auffassen, weiter aber konnten Cerebrin und Homocerebrin homologe Körper sein. Da es nicht gelang, Cerebrin durch wasserentziehende Mittel in Homocerebrin überzuführen, und die Versuche, Cerebrin aus Homocerebrin darzustellen, gleichfalls ohne Resultat blieben, so gewinnt die Annahme der Homologie an Wahrscheinlichkeit; freilich kann diese Frage nur durch eingehendes Studium 'der Spaltungsprodukte von Cerebrin und Homocerebrin entschieden werden.

Eine andere Frage ist: Sind Cerebrin und Homocerebrin ursprüngliche Bestandtheile des Gehirns, oder Zersetzungsprodukte des Protagons? Von allen Forsehern hat nur Diaconow durch Kochen mit wässrigem Baryt aus Protagon eine Substanz erhalten, welche einige Aehnlichkeit mit Cerebrin besitzt. „Sie ist ganz neutral, quillt in heissem Wasser, löst sich in heissem Alkohol und Aether, nicht aber in kaltem auf. Beim Verbrennen hinterlässt dieselbe keine Asche, sie enthält keinen Phosphor, weiteres Kochen mit

1) Dies. Journ. [2] 17, 232. 
Barytwasser verändert die Substanz nicht. Mit Schwefelsäure behandelt reducirt dieselbe Kupferoxyd."

Obgleich das Cerebrin nicht alle Eigenschaften besitzt, wie sie Diaconow beschreibt, denn Cerebrin ist in Aether unlöslich, und wird durch Kochen mit Barytwasser verändert, so kann es doch Cerebrin gewesen sein, welches der genannte Forscher in Händen gehabt hat; eben so gut aber auch Homocerebrin oder Enkephalin, welche jene Eigenschaften mit Cerebrin theilen. Der Umstand aber, lass Diaconow das von ihm dargestellte Protagon für ein Gemenge von Cerebrin uud Lecithin hielt, macht die Beurtheilung und Entscheidung dieser Frage überflüssig.

Liebreich $\left.{ }^{1}\right)$ hat nach 24 stündigem Kochen des Protagons mit Barytwasser weiter nichts als die Zersetzungsprodukte des Lecithins gefunden. Ebenso kann weder Cerebrin noch Homocerebrin mit derjenigen Substanz identisch sein, welche derselbe Forscher ${ }^{2}$ ) durch Kochen von Protagon mit ganz verdünnter Salzsäure erhielt, eine Substanz, die ähnlich wie das Protagon in Nadeln krystallisirt; denn diese besitzt die Eigenschaft, in alkoholischer Lösung dem directen Sonnenlichte ausgesetzt, sich unter Abscheidung eines braunen Pulvers zu zersetzen. Schon diese Angaben früherer Forscher sprechen dafür, dass Cerebrin und Homocerebrin von Protagon nicht abstammen, und die vorläufige Mittheilung von Baeyer und Liebreich ${ }^{3}$ ), wonach Protagon durch Spaltung reichliche Mengen von Zucker und die Zersetzungsprodukte des Lecithins liefert, ist geeignet, diese Ansicht zu unterstützen. Von grösserer Wichtigkeit für die Entscheidung dieser Frage sind die Produkte der Zersetzung, welche Gamgee nnd Blankenhorn ${ }^{+}$) durch Kochen von Protagon mit Aether erhielten: "Zweimal umkrystallisirtes Protagon wurde in einer mit Rückflusskühler versehenen Kochflasche

1) Liebreich, Ann. Chem. Pharm. 134, 34.

2) Liebreich, Ann. Chem. Pharm. 134, 38.

3) Baeyer und Liebreich, Virchow's Archiv 39, 183.

4) Gamgee und Blankenhorn, Hoppe-Seyler's Zeitschr. f. physiolog. Chem. 3, 280. 
mit einem grossen Quantum Aether übergossen, dasselbe im Wasserbade 15 Stunden im Kochen erhalten, erkalten gelassen, filtrirt und die gelöste Substanz bei $45^{0}$ aus Weingeist umkrystallisirt. Der Körper scheidet sich in körnigen Massen aus, die indess unter dem Mikroskop von Protagon verschiedenes Aussehen besitzen. Nadeln wurden nicht erhalten." Die Resultate der Analysen dieser Substanz lieferten Zahlen, welche ganz bedeutend von den Durchschnittszahlen der Analysen vọ Cerebrin und Homocerebrin abweichen.

2 mal umkryst. mit

Aether behand. Protagon.

\begin{tabular}{llr}
$\mathrm{C}$ & $=1$. & \multicolumn{1}{c}{2.} \\
$\mathrm{H}$ & $=13,2$ & 63,1 \\
$\mathrm{~N}$ & $=-$ & 9,3 \\
$\mathrm{P}$ & $=0,72$ & -
\end{tabular}

Cerebrin. Homocerebrin.

70,06

11,595

2,23 .

Diese auffallende Differenz kann nicht durch eine Verunreinigung des Aetherproduktes veranlasst sein, denn dasselbe war durch Unkrystallisiren aus Alkohol gereinigt worden, ebenso bleibt der Gedanke, dass eine Zersetzung des gebildeten Cerebrins oder Homocerebrins stattgefunden, ausgeschlossen, da beide durch Kochen mit Aether nicht im geringsten angegriffen werden. Andere Produkte wurden bei dieser Operation nicht erhalten, oder waren in kaltem Aether löslich und kommen deshalb nicht in Betracht. Somit ist der Schluss gerechtfertigt: Cerebrin und Homocerebrin sind keine Zersetzungsprodukte des Protagons, folglich ursprüngliche Bestandtheiln des Gehirns, wenigstens so lange es nicht gelingt, aus demselben neue, bis jetzt noch nicht bekamnte Stoffe zu isoliren, welche Verbindungen von Cerebrin oder Homocerebrin mit anderen Körpern darstellen. Das indifferente Verhalten von Cerebrin und Homocerebrin spricht nicht dafür, dass solche Verbindungen existiren.

III. Enkephalin.

Die Hauptmenge des Enkephalins war in dem mit D bezeichneten Gemenge von Homocerebrin und Enkephalin enthalten, eine geringe Menge in der Substanz M, ein weiJourual f. prakt. Chemie [2] Bd. 24. 
338 Parcus: Ueber einige neue Gehirnstoffe.

terer Theil fand sich, ebenfalls mit Homocerebrin gemeugt, in den Aetherauszügen, welche durch fortgesetzte Extraction des Rohcerebrins nach Entfernung des Cholesterins erhalten worden waren. Man wird sich erinnern, dass diese Substanz D die löslicheren Theile der Substanz M enthielt. Ich konnte daher erwarten, dass Enkephalin in Alkohol viel löslicher sei, als Homocerebrin. Leider bestätigte jedoch der Versuch diese Vermuthung nicht. Wurde eine heisse, alkoholische Lösung der Substanz D abgekühlt, so erhielt man in dem weissen, flockigen Niederschlage ein Gemenge, welches zu ziemlich gleichen Theilen aus Homocerebrin und Enkephalin bestand. Die Mutterlauge hiervon lieferte, auf ein Viertel des ursprünglichen Volums eingedampft und abgekühlt, einen Niederschlag, welchier reichliche Mengen von ziemlich reinem Enkephalin ausschied. Die Mutterlauge dieser letzten Ausscheidung ergab dagegen weiter eingedampft wieder ein Gemenge von Homocerebrin und Enkephalin. Da Homocerebrin und Enkephalin sich in Alkohol viel löslicher zeigten als in Aceton, benutzte ich zur genaueren Trennung letztere Flüssigkeit. Das Enkephalin wurde sodamı durch häufiges Umkrystallisiren gereinigt. Die geringe Menge gestattete es nicht, viele Versuche mit diesem Körper anzustellen. Die Resultate der Analysen, bei welchen der höhere Stickstoffgehalt von 3,09\% hervorgehoben zu werden verdient, sind oben angeführt.

Wie bezüglich seiner Löslichkeit, so steht es auch in seinen sonstigen Eigenschaften dem Homocerebrin näher als dem Cerebrin und vermag, von Alkohol aufgenommen, eine Gallerte zu bilden. Ganz reines Enkephalin scheidet sich aus verdunstenden Lösungen in leicht gekrümmten, schönen Blättchen aus. Geringe Verunreinigungen beeinträchtigen diese Ausscheidung. Aus Aceton fällt es beim raschen Abküblen in weissen, körnigen Massen zu Boden, welche beim Trocknen stark einschrumpfen.

Dampft man die Lösung auf dem Wasserbade ein, so hinterbleibt eine spröde, glasige Masse von gelber Farbe. Trocken erhitzt zersetzt es sich bei $125^{n}$; wird es weiter erhitzt, so verwandelt es sich bei $150^{\circ}$ in eine braune Flüs- 
sigkeit, welche sich wie geschmolzenes Cerebrin verhält. Am meisten unterscheidet es sich von Cerebrin und Homocerebrin durch sein Aufquellen in heissem Wasser, mit dem es einen vollständigen Kleister bildet, ein Zustand, in dem es auch nach dem Erkalten verharrt. Kocht man es kurze Zeit mit Salzsäure, so erhält man eine alkalisches Kupferoxyd reducirende Lösung. Der Rückstand enthält dann immer noch von den oben beschriebenen Blättchen, wird aber durch weiteres Kochen mit Salzsäure vollständig zersetzt und in einen amorphen Körper verwandelt. Bezüglich der Herkunft des Enkephalins bin ich zu keinem Resultat gelangt, möchte aber annehmen, dass es aus Cerebrin oder Homocerebrin hervorgegangen sei, wofür die geringe Menge des vorgefundenen Materials, sowie das Erscheinen von Blättchen nach kurzem Kochen von Cerebrin oder Homocerebrin mit überschüssigem Barytwasser oder Salzsäure - letztere das geeignetere Mittel - zu sprechen scheint.

Das Auftreten anderer Zersetzungsprodukte erschwert die Isolirung der bei der Zersetzung von Cerebrin oder Homocerebrin entstehenden Blättchen so ausserordentlich, dass ich von einer Reinigung abstand.

Die Resultate vorliegender Untersuchung sind folgende:

Es ist mir gelungen nachzuweisen, dass dem Cerebrin auch nach fortgesetztem Umkrystallisiren aus Alkohol der Stickstoff nicht entzogen werden kann; dass ferner diese Substanz Eigenschaften besitzt, welche ausserordentlich verschieden sind von den Eigenschaften, welche dem von Müller als Cerebrin bezeichneten Körper zukommen; dass endlich das früher beschriebene Cerebrin ein Gemenge dreier einander nahe stehender, indifferenter, stickstoffhaltiger Körper ist, welche ich Cerebrin, Homocerebrin und Enkephalin benannt habe; Körper, deren Analysen nach erfolgter Trennung und Reinigung im Mittel folgende Zahlen lieferten:

$$
\begin{array}{lccc} 
& \text { Cerebrin. } & \text { Homocerebrin. } & \text { Fnkephalin. } \\
\mathrm{C}=69,08 & \mathbf{7 0 , 0 6} & 68,40 \\
\mathrm{H}=11,47 & \mathbf{1 1 , 5 9 5} & 11,60 \\
\mathrm{~N}= & 2,13 & 2,23 & 3,09 \\
\mathrm{O}=17,32 & 16,115 & 16,91
\end{array}
$$


340 Kolbe: Blumenlese modern-chem. Aussprüche.

Das weitere Studium derselben behalte ich mir vor.

Der Nachweis verschiedener Cerebrine, als einer Gruppe im Gehirn vorhandener, gut charakterisirter Körper lässt vermuthen, dass die Reihe der Gehirnstoffe durch die bis jetzt vorliegenden Resultate der Forschung ihren Abschluss noch nicht gefunden hat, und dass eine sorgfältige Untersuchung dieses Theils des thierischen Organismus demjenigen. eine reiche Ausbente verspricht, welcher die Schwierigkeiten nicht scheut, die sich seinem Studium entgegenstellen.

Vorliegende Untersuchungen wurden in der chemischen Abtheilung des physiologischen Instituts zu Leipzig auf Anregung des Hrn. Prof. Dr. Drechsel ausgeführt. Das stets bewiesene warme Interesse, wie der vielfache Rath, dessen ich mich von seiner Seite zu erfreuen hatte, verpflichten mich zu bleibendem Danke.

\section{Blumenlese modern-chemischer Aussprüche.}

(Mitgetheilt von H. Kolbe.)

16.

„Das Kohlenoxyd, CO, ist das den Kohlensäurederivaten "gemeinschaftliche Radikal, und wird als solches Carboxyl "genannt" (W islic en us, Lehrbuch der org. Chemie, S. 144).

Die Studirenden, welche Wislicenus Lehrbuch zur Hand nehmen, werden nicht wissen, was sie glauben sollen, wenn sie sonst überall lesen und gelernt haben, dass der Name Carboxyl für das Radikal: $\mathrm{COOH}$ gebraucht wird. Jener Satz würde verständlich sein, wenn er so lautete: „Das Kohlenoxyd CO führt als Radikal den Namen Carbonyl. Ich nehme mir die Freiheit, dem Kohlenoxyd den Namen Carboxyl zu geben, obschon dieser von allen anderen Chemikern für das Radikal von der Zusammensetzung $\mathrm{COOH}$ gebraucht wird." 